\title{
Spatially Resolved Proteomic Analysis of the Lens Extracellular Diffusion Barrier
}

\author{
Zhen Wang, Lee S. Cantrell, and Kevin L. Schey \\ Department of Biochemistry, Vanderbilt University, Nashville, Tennessee, United States
}

Correspondence: Kevin L. Schey, Mass Spectrometry Research Center, Vanderbilt University, 465 21st Ave. So., Suite 9160 MRB III, Nashville, TN 37232-8575, USA;

kevin.schey@vanderbilt.edu.

Received: March 8, 2021

Accepted: May 5, 2021

Published: September 23, 2021

Citation: Wang Z, Cantrell LS, Schey KL. Spatially resolved proteomic analysis of the lens extracellular diffusion barrier. Invest Ophthalmol Vis Sci. 2021;62(12):25.

https://doi.org/10.1167/iovs.62.12.25
Purpose. The presence of a physical barrier to molecular diffusion through lenticular extracellular space has been repeatedly detected. This extracellular diffusion barrier has been proposed to restrict the movement of solutes into the lens and to direct nutrients into the lens core via the sutures at both poles. The purpose of this study is to characterize the molecular components that could contribute to the formation of this barrier.

MEтнорs. Three distinct regions in the bovine lens cortex were captured by laser capture microdissection guided by dye penetration. Proteins were digested by Lys $\mathrm{C}$ and trypsin. Mass spectrometry-based proteomic analysis followed by gene ontology and protein interaction network analysis was performed.

Results. Dye penetration showed that fiber cells first shrink the extracellular spaces of the broad sides followed by closure of the extracellular space between narrow sides at a normalized lens distance (r/a) of 0.9 . Accompanying the closure of extracellular space of the broad sides, dramatic proteomic changes were detected, including upregulation of several cell junctional proteins. AQP0 and its interacting partners, Ezrin and Radixin, were among a few proteins that were upregulated, accompanying the closure of extracellular space of the narrow sides, suggesting a particularly important role for AQP0 in controlling the narrowing of the extracellular spaces between fiber cells. The results also provided important information related to biological processes that occur during fiber cell differentiation such as organelle degradation, cytoskeletal remodeling, and glutathione synthesis.

Conclusions. The formation of a lens extracellular diffusion barrier is accompanied by significant membrane and cytoskeletal protein remodeling.

Keywords: lens fiber cells, extracellular diffusion barrier, laser capture microdissection, quantitative mass spectrometry
$\mathrm{T}$ achieve its normal function as an optical element and to maintain its transparency, the lens has evolved a unique structure that lacks light-scattering elements, including blood vessels and cellular organelles. ${ }^{1}$ In addition, the terminally differentiated fiber cells are closely packed with the extracellular space smaller than the wavelength of the light to reduce light scattering. ${ }^{1}$ In the absence of a blood supply, this large tissue cannot rely on simple diffusion to deliver nutrients to the center of the lens. It has been demonstrated that the lens establishes a microcirculation system to deliver nutrients, remove wastes, maintain ionic homeostasis, and control the volume of the fiber cells. ${ }^{1-4}$ Based on the microcirculation system model, a circulating current, carried primarily by $\mathrm{Na}^{+}$ions, directs nutrients extracellularly to the center of the lens at both poles via the sutures. $\mathrm{Na}^{+}$ ions along with water and metabolites in the center of the lens diffuse toward the lens equator intercellularly (i.e., from cell to cell across cell membranes) and flow toward the lens surface through gap junctions. The formation of the microcirculation system relies on spatially distinct distributions of ion channels and transporters, including highly concentrated activity of sodium-potassium pumps in the anterior epithelial cells ${ }^{5,6}$ and a high gap junction coupling conduc- tance at the equator. ${ }^{7}$ The circulating current at the surface of the lens has been confirmed experimentally. ${ }^{8}$ Magnetic resonance imaging has allowed visualization of fluid fluxes of heavy water $\left(D_{2} \mathrm{O}\right)$ that are directed into the lens at the poles and then move circumferentially toward the equator in the lens cortex. ${ }^{9}$ This study also identified a zone of restricted extracellular space diffusion. ${ }^{9}$ A physical barrier to extracellular space diffusion has been reported in lenses from different species, ${ }^{10,11}$ which is consistent with the observed reduction of extracellular space between fiber cells to avoid light scattering that has been confirmed by electron microscopy. ${ }^{12}$ This extracellular diffusion barrier has been proposed to restrict the movement of solutes into the lens and acts to direct nutrients into the lens core via the suture at both poles.?

The molecular mechanism that controls closing of the extracellular space and formation of the extracellular diffusion barrier has not been studied. Tight junctions are normally believed to control paracellular permeability and provide a diffusion barrier. ${ }^{13}$ Occluded zones similar to tight junctions were described between lens fiber cells in an early electron microscopy study. ${ }^{12}$ Several well-known tight junction proteins can be detected in lens fiber cells such as ZO-1, 
junction adhesion molecule 3 (JAM3), and coxsackie and adenovirus receptor (Cxadr). ${ }^{14,15}$ However, due to lack of core proteins of tight junction occludins or claudins, tight junctions were reported to be absent in lens fiber cells. ${ }^{14-16}$ In the absence of claudins, lens fiber cells express lens fiber membrane intrinsic protein (LIM2), a member of the PMP-22/EMP/MP20/claudin family that shares a tetraspanin topology and the WGLWCC signature sequence in the first extracellular loop. ${ }^{17}$ The precise function of LIM2 is unknown, but an adhesive function ${ }^{18}$ and a role in fiber cell junction formation and organization have been reported. ${ }^{19}$ Another unique structure present in lens fiber cells is the square array junction. ${ }^{20-22} \mathrm{AQP0}$ and its cleavage products were found to be enriched in square array junctions. ${ }^{20,21}$ Previous electron microscopy data suggested that the reduction of lenticular intercellular space was correlated with the formation of square array and membrane undulation..$^{20,21}$ Thus, square arrays might potentially drive the formation of complicated membrane interdigitations and serve to maintain an extremely narrow extracellular space. ${ }^{20,22}$ In addition, it has been reported that gap junction plaques on the broad side of the outer fiber cells could restrict penetration of larger solutes through extracellular space.?

The purpose of this study was to investigate proteins that could regulate and control the closure of lens extracellular spaces. Previous studies mainly relied on immunohistochemistry and confocal and electron microscopy. ${ }^{10,11,20,22}$ Mass spectrometry-based quantitative proteomic analysis is a powerful discovery approach to examine global proteomic dynamics. We hypothesize that an in-depth spatially resolved proteomic study will reveal proteins and pathways that could be involved in controlling extracellular space and diffusion. In this article, a dye penetration experiment defined three distinct regions within less than $1.5 \mathrm{~mm}$ distant from the lens capsule to inner cortex in the equatorial region of bovine lenses. These three regions were then isolated by laser capture microdissection, and proteome changes across three regions were studied with high-throughput quantitative mass spectrometry. Our results showed that the extracellular diffusion barrier is formed in a stepwise manner accompanied with dramatic changes in fiber cell junctional proteins, membrane, and cytoskeletal proteins.

\section{Materials AND Methods}

\section{Dye Penetration and Confocal Imaging}

Bovine lenses were extracted from fresh cow eyes (Light Hill Meats, Lynnville, TN, USA) and incubated in M199 medium (Sigma, St. Louis, MO, USA) that contained $1 \mathrm{mg} / \mathrm{mL}$ Texas Red-dextran (10,000 MW, Lysine Fixable; Thermo Fisher Scientific, Rockford, IL, USA) at $37^{\circ} \mathrm{C}$ for 6 to 24 hours. All lenses used in this study were from cows around 2 years old. After incubation, lenses were rinsed with M199 media and fixed in $50 \mathrm{~mL} 2 \%$ paraformaldehyde containing $0.01 \%$ glutaraldehyde for 4 days. The lenses were washed three times with PBS for 20 minutes and then cryoprotected with $10 \%$ sucrose in PBS for 1 day at $4{ }^{\circ} \mathrm{C}, 20 \%$ sucrose for 5 hours at room temperature and 30\% sucrose for 1 hour at room temperature and an additional 2 days at $4^{\circ} \mathrm{C}$. For sectioning, lenses were soaked in Tissue-Tek OCT (Sakura Finetek, Torrance, CA, USA) compound for 20 minutes and mounted in equatorial orientation on chucks and frozen in liquid nitrogen. Lenses were sectioned to a 30- $\mu \mathrm{m}$ thickness using a CM 3050 Cryostat (Leica CM 3050S; Leica
Microsystems, Inc., Bannockburn, IL, USA). The sections were transferred to glass slides and washed three times in PBS before mounting on glass slides with mounting media (Vector Laboratories, Burlingame, CA, USA). Confocal imaging was performed using a Zeiss LSM 710 Confocal Microscope (Carl Zeiss AG, Oberkochen, Germany) with a $20 \times$ objective. Airyscan imaging was performed with a confocal laser scanning microscope (Zeiss LSM 880; Carl Zeiss AG) with a Plan-Apochromat $63 \times / 1.46$ Oil Corr M27 objective.

\section{Laser Capture Microdissection}

Bovine lenses were incubated with Texas Red-labeled dextran for 18 hours as described above. Lenses were rinsed with M199 media and frozen immediately at $-80^{\circ} \mathrm{C}$. Then, 1- $\mu$ m-thick fresh-frozen sections were obtained using the same cryostat as mentioned above. Four lenses from four different animals were used to generate four biological replicates. The lenses were sectioned from the anterior pole, and 10 equatorial sections were collected from each lens at around a $4.2-\mathrm{mm}$ distance from the anterior pole. The sections were collected on PEN membrane slides (Carl Zeiss, Munich, Germany) and stored at $-80^{\circ} \mathrm{C}$. Before laser capture microdissection (LCM), sections were dehydrated with $75 \%$ ethanol for 1 minute and 100\% ethanol for 1 minute and air dried. The LCM procedure was conducted using a PALM UV Laser MicroBeam laser capture microdissection system (Carl-Zeiss AG). Tissue regions were selected based on the measured distance from the lens capsule. Depending on the regions, tissue was cut at an energy level of 38 to 43 and catapulted into an $0.2-\mathrm{mL}$ Eppendorf tube cap containing $25 \mu \mathrm{L}$ HPLC-grade water. Normally, 3 to $4 \times 10^{6} \mu \mathrm{m}^{2}$ tissue can be collected in one cap, and 1.6 to $2.8 \times 10^{7}{\mu m^{2}}^{2}$ tissue was collected from each region in total. Three different regions were captured based on confocal images from the dye penetration experiment. The first sample captured contains fiber cells 50 to $350 \mu \mathrm{m}$ from the lens capsule followed by capture of a region 450 to $800 \mu \mathrm{m}$ from the capsule for the second sample and finally a region 950 to $1250 \mu \mathrm{m}$ from the capsule for the third sample. The three regions captured were named outer cortex 1 (OC1), outer cortex 2 (OC2), and inner cortex (IC). The IC region corresponds to the start of the barrier region. Five samples (collected in 0.2-mL Eppendorf tube caps) from each region were pooled into one tube and dried in a speedvac and deemed ready for further processing.

\section{Sample Preparation and LC-MS/MS Analysis}

In total, $20 \mu \mathrm{L} 100 \mathrm{mM}$ ammonium bicarbonate containing $2 \mathrm{M}$ urea and $10 \mathrm{mM}$ tris(2-carboxyethyl)phosphine (TCEP) (pH 8.0) was added to each sample, and the samples were incubated at $25^{\circ} \mathrm{C}$ for 45 minutes. Then, $2 \mu \mathrm{L} \mathrm{2-}$ chloroacetamide $(500 \mathrm{mM})$ was added to each sample to alkylate free cysteines. The samples were incubated at $25^{\circ} \mathrm{C}$ for 45 minutes. The samples were then centrifuged at 20,000 $\times g$ for 5 minutes, and the supernatant was carefully separated from the pellets (not visible). The remaining pellets were washed with $20 \mu \mathrm{L} 100 \mathrm{mM}$ ammonium bicarbonate and $2 \mathrm{M}$ urea ( $\mathrm{pH}$ 8.0) for another three times, and the supernatants were pooled as the soluble fraction (SF). The remaining pellets were called the insoluble fraction (ISF). Then, $5 \mu \mathrm{L}$ of each SF was diluted seven fold in water and used for a Bradford protein assay (Thermo Fisher Scientific). 
In total, $15 \mu \mathrm{g}$ total protein from each soluble fraction was used for enzyme digestion, and $2 \mathrm{M}$ urea in $100 \mathrm{mM}$ ammonium bicarbonate was added to each sample to regulate the final volume to $100 \mu \mathrm{L}$. Then, $100 \mu \mathrm{L} 100 \mathrm{mM}$ ammonium bicarbonate was added to each sample to reduce the urea concentration to $1 \mathrm{M}$. The proteins were digested by endoproteinase Lys $C$ (1:25 enzyme to protein ratio) for 2 hours at $37^{\circ} \mathrm{C}$, followed by trypsin digestion (1:25 enzyme to protein ratio) at $37^{\circ} \mathrm{C}$ for 6 hours. For each insoluble fraction, $5 \mu \mathrm{L}$ of acetonitrile (ACN) and $45 \mu \mathrm{L}$ of $100 \mathrm{mM}$ ammonium bicarbonate were added to each pellet. The samples were then digested by Lys $\mathrm{C}$ and trypsin as above. A protein assay was not done for ISF samples due to the low amount of protein present. The amount of enzyme used for digestion of the ISF was based on the total proteins measured in SF of the corresponding sample (1:200 enzyme to SF protein ratio). ISF samples were dried in a speedvac after digestion and reconstituted in $0.1 \%$ formic acid for StageTip cleaning. SF samples were acidified and cleaned by StageTips as described by Rappsilber et al. ${ }^{23}$ The StageTips were made by placing a small portion of Empore solid-phase extraction disks (3 M) in a $200-\mu \mathrm{L}$ pipette tip. An additional $1 \mathrm{mg}$ C18

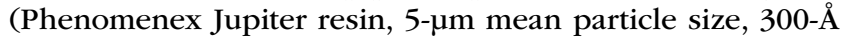
pore size) was loaded into the tip for SF samples, and $0.5 \mathrm{mg}$ C18 was loaded into the tip for ISF samples. Peptides were eluted in $70 \%$ ACN containing $0.1 \%$ formic acid. All samples after StageTip cleaning were dried in a speedvac and reconstituted in $0.1 \%$ formic acid. The final concentration of the SF was $0.1 \mu \mathrm{g} / \mu \mathrm{L}$. The amount of proteins in the ISF was estimated to be around 1/15th of the SF based on base peak intensities in LC-MS/MS runs. Therefore, ISF samples were reconstituted in $0.1 \%$ formic acid, and the volumes were $1 / 15$ th of the volume used for reconstituting corresponding soluble fractions. For each sample, $500 \mathrm{ng}$ total protein was used for LC-MS/MS analysis.

For LC-MS/MS analysis, samples were separated on a one-dimensional fused silica capillary column $(200 \mathrm{~mm} \times$ $100 \mu \mathrm{m})$ packed with Phenomenex Jupiter resin (3- $\mu \mathrm{m}$ mean particle size, $300-\AA$ pore size) coupled with an UltiMate 3000 RSLCnano system (Thermo Scientific, San Jose, CA). A 76-minute gradient was performed, consisting of the following: 2 to 72 minutes, $2 \%$ to $40 \% \operatorname{ACN}(0.1 \%$ formic acid) and 75 to $78 \mathrm{~min}, 45 \%$ to $90 \% \mathrm{ACN}$ ( $0.1 \%$ formic acid) balanced with $0.1 \%$ formic acid. The flow rate was $350 \mathrm{~nL} / \mathrm{min}$. The eluate was directly infused into a $\mathrm{Q}$ Exactive Plus instrument (Thermo Scientific) equipped with a nanoelectrospray ionization source. The data-dependent instrument method consisted of MS1 acquisition $(\mathrm{R}=70,000)$ from $m / z 350$ to 1600, using an MS AGC target value of $3 \mathrm{e} 6$ and maximum ion time of $60 \mathrm{~ms}$, followed by up to $20 \mathrm{MS} / \mathrm{MS}$ scans $(\mathrm{R}=$ 17,500) of the most abundant ions detected in the preceding MS scan. The MS2 AGC target value was set to 5e4, with a maximum ion time of $100 \mathrm{~ms}$; higher energy collision dissociation (HCD) collision energy was set to 27; and dynamic exclusion was set to 10 seconds.

\section{Data Analysis}

The raw data have been deposited to the PRIDE Archive with a data set identifier (PXD025337). Raw LC-MS/MS data were loaded into MaxQuant software (version 1.6.6.0; The Max Planck Institute of Biochemistry, Munich, Germany) ${ }^{24}$ and searched against a canonical bovine database with 23,847 sequences (Proteome ID: UP000009136, downloaded April 13, 2021) with contaminant proteins included using the Andromeda search algorithm within MaxQuant. ${ }^{25}$ The search was performed with trypsin specificity with a maximum of two missed cleavage sites. Methionine oxidation, asparagine deamidation, and protein N-terminal acetylation were variable modifications (up to two modifications allowed per peptide); cysteine was assigned a fixed carbamidomethyl modification. Precursor mass tolerance was set at $4.5 \mathrm{ppm}$; the minimum peptide length was 6 amino acids, and maximum peptide mass was $4600 \mathrm{Da}$. A false discovery rate (FDR) of $1 \%$ was applied for both peptide and protein filtering, and at least two unique plus razor peptides (the peptides assigned to the protein group with the largest number of total peptides identified) were used for protein identification. Quantification settings were as follows: requantify with a second peak-finding attempt after protein identification has completed and match full MS1 peaks between runs within a 0.7 -minute retention time window. The label-free quantitation (LFQ) algorithm in MaxQuant $^{24}$ was used for protein quantitation. Only razor and unique peptides were used for protein-level quantitation. LFQ was performed between samples OC1 and OC2 as well as between OC2 and IC. Considering dramatic proteome changes, protein quantification in ISF and SF was done separately, and LFQ normalization was not done across ISF and SF.

The LFQ intensities of proteins from the MaxQuant searches were imported into Perseus (V1.5.2.3; The Max Planck Institute of Biochemistry, Munich, Germany). ${ }^{26}$ Contamination and reverse identifications were filtered, and the remaining protein LFQ intensities were $\log 2$ transformed. For quantification, only proteins that were present in at least $75 \%$ of replicates in at least one region were used, and missing values were imputed with random numbers from a normal distribution $(1.8,0.3)$. Welch test in Perseus was used for identification of significantly changed proteins between regions $(P<0.05)$. Pearson correlations were calculated though the multiscatter plot function in Perseus. Hierarchical clustering was completed based on Euclidean distance. Volcano plots were done in Perseus with more stringent parameters (FDR $=0.01$ and $S 0=0.5$ ) for visualizing more significantly changed proteins between regions. To improve ontology assignments, bovine proteins were mapped to human orthologs with db_to_db_blaster.py script available at https://github.com/ pwilmart/PAW_BLAST. The query database was the previously used canonical bovine fasta file, and hit database was the reviewed human proteome fasta (UP000005640, downloaded March 12, 2021). Only high-quality matches were used for ortholog mapping in R. Enriched Gene Ontology (GO) terms and pathways were determined using Fisher exact test with a Benjamini-Hochberg FDR of 0.05 and total quantified proteins as the background. Protein-protein network analyses were generated by STRING $11.0^{27}$ using medium or high confidence.

\section{Results}

\section{Characterization of the Extracellular Diffusion Barrier in Bovine Lenses}

Dye penetration images (Fig. 1) show that Texas Redlabeled dextran $(\sim 10 \mathrm{kDa})$ penetrated the lens through the extracellular space, and two distinct regions in the outer cortex were observed. From the capsule to 350 to $400 \mu \mathrm{m}$, the dye easily penetrated the lens, and dye signal in this 

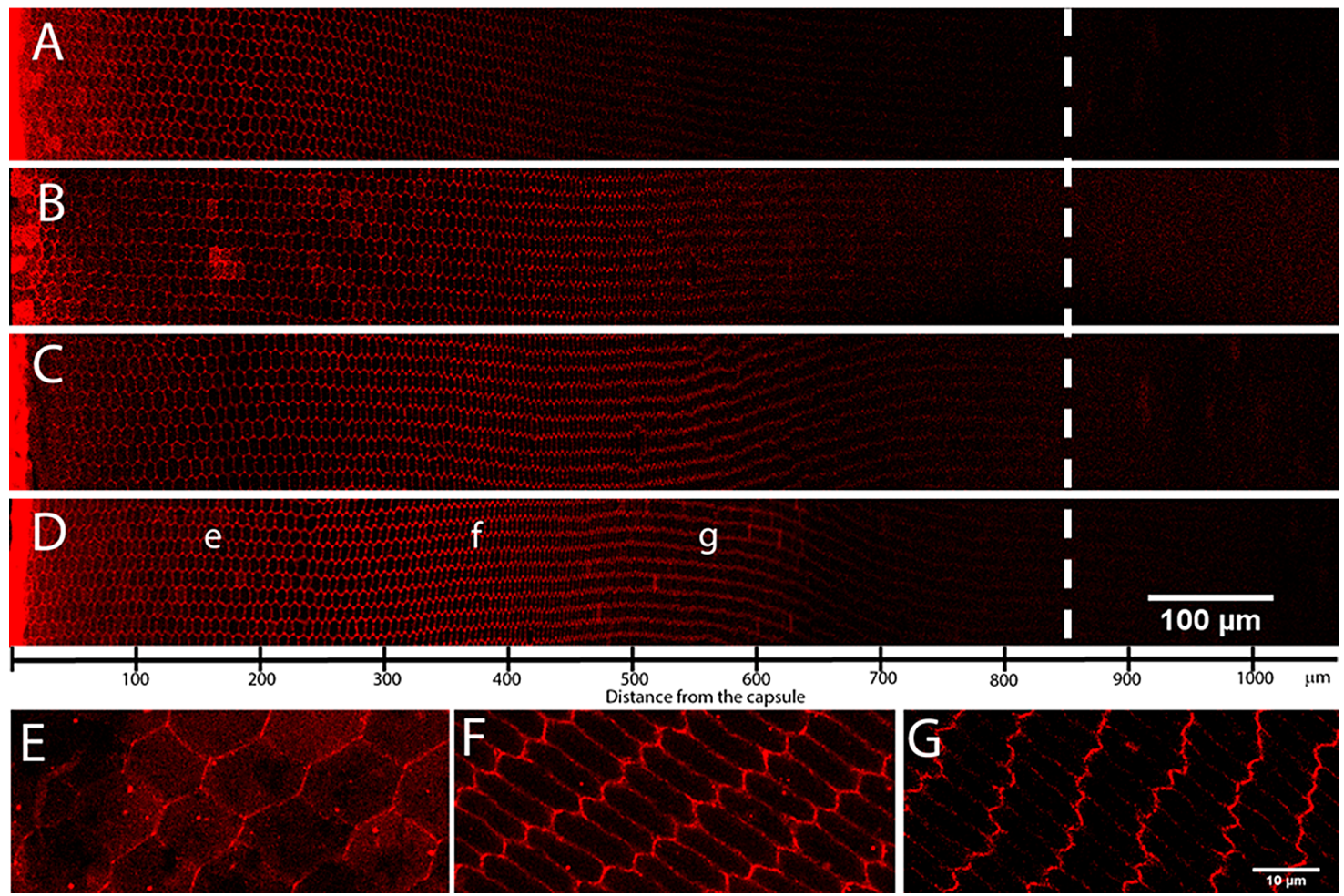

Figure 1. Localization of the extracellular diffusion barrier. Bovine lenses were dissected from fresh cow eyeballs and incubated with lysine fixable Texas Red-dextran for various periods of time (6-24 hours). The lenses were then fixed and imaged by confocal microscopy (A-D). Dye incubation time was 6 hours (A), 9 hours (B), 18 hours (C), and 24 hours (D). The result showed the dye penetrated the lens through extracellular spaces, and farther from the capsule into the lens, dye penetration through the short sides of the fiber cells was inhibited. Regardless of incubation time, at 850 to $900 \mu \mathrm{m}$, dye penetration through extracellular spaces stopped. Airyscan images for corresponding regions in $\mathbf{D}$ are shown in $\mathbf{E}-\mathbf{G}$ (corresponding to area labeled by $\mathbf{e}-\mathbf{g}$, respectively) showing the fiber cell morphology and dye penetration changes in outer cortex of the lens. The dotted line indicates the position with normalized lens distance of 0.9 .

region did not change with longer incubation times. Fiber cells in this region have regular hexagonal shapes. Further into the lens, fiber cells became more compact, and dye penetration between the broad sides of the fiber cells was inhibited and penetration through the narrow sides remained. Dye penetration through all extracellular spaces completely stopped at 850 to $900 \mu \mathrm{m}$ from the capsule. Using three different lenses, the average normalized lens distances $\mathrm{r} / \mathrm{a}$ (where a represents the lens radius and $\mathrm{r}$ represents the distance from the lens core to the region of interest) for dye penetration were $0.919 \pm 0.007$ for a 6-hour incubation, $0.913 \pm 0.004$ for a 9-hour incubation, $0.896 \pm 0.014$ for an 18-hour incubation, and $0.897 \pm 0.006$ for a 24 -hour incubation. The normalized distances for 18-hour and 24-hour incubated lenses were not statistically different, indicating a barrier to extracellular diffusion starts at an average normalized lens distance of 0.9 in the inner cortex of the lens.

\section{LFQ Quantification Shows Dramatic Proteome Changes in Lens Cortex}

Based on dye penetration experiments, three different regions in lens cortex were isolated by LCM. Note that the first $50 \mu \mathrm{m}$ from the capsule was not captured to avoid the lens epithelium. The OC1 region $(50-350 \mu \mathrm{m})$ represents peripheral fiber cells. In this region, the Texas Redlabeled dextran can penetrate through extracellular spaces surrounding the entirety of the fiber cells. The OC2 region
(450-800 $\mu \mathrm{m})$ represents the region where dye penetration through the broad sides of the fiber cells was inhibited. The IC region (950-1250 $\mu \mathrm{m})$ represents the region where dye penetration is completely stopped. After LCM, the samples were separated into soluble and insoluble fractions and analyzed separately to comprehensively characterize soluble proteins and membrane and membrane-associated protein expression by LFQ using MaxQuant.

Data from three regions were initially searched together through MaxQuant, and proteomes in different regions were found to be significantly altered. Normalization for quantitative proteomic studies relies on a majority of the proteome not being altered. The normalization step performed by MaxQuant has been shown to provide reliable protein quantification when one-third of the proteome was changing ${ }^{24}$; however, to avoid potential effects of dramatic proteome changes on normalization, comparisons were only done between OC1 and OC2 regions or between OC2 and IC regions. The OC1 region was not compared to the IC region. In total, 1408 proteins and 799 proteins were identified in the ISF and SF samples of OC1 and OC2, respectively. In total, 1076 proteins and 552 proteins were identified in ISF and SF of OC2 and IC, respectively. Venn diagrams showing the overlaps in protein identification among different samples are shown in Figures 2A and 2B. Even though Venn diagrams show significant overlaps in protein identification in different regions, a trend of a decrease in the number of proteins identified can be seen comparing OC2 to OC1 or IC to OC2. When protein signal intensities were compared, 

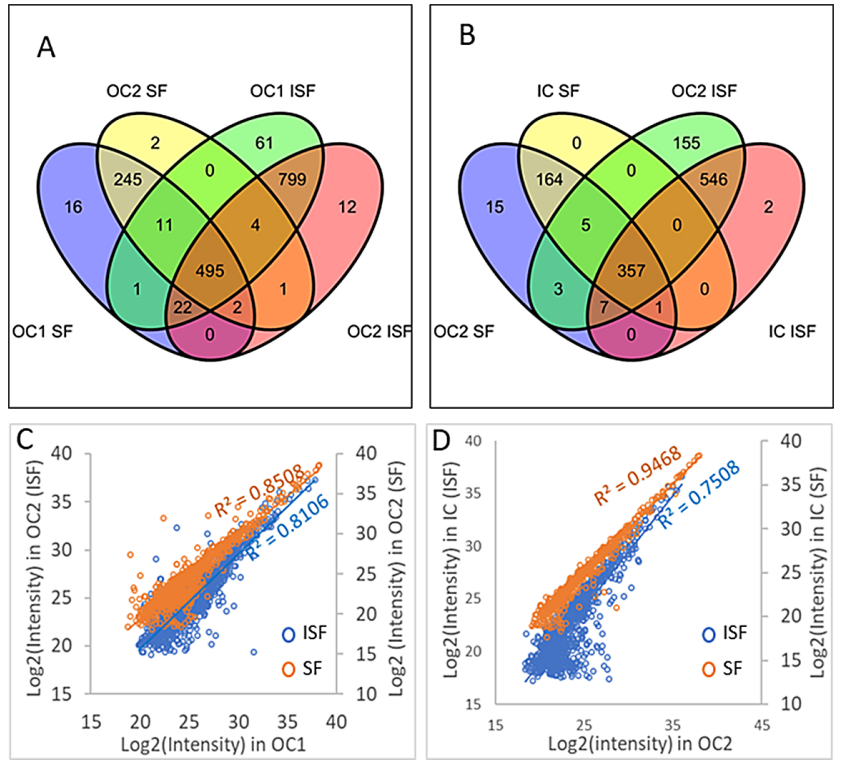

Figure 2. Venn diagrams and scatterplots. Three different regions of bovine lenses were isolated by LCM. The overlaps of the lists of protein IDs detected by LC-MS/MS in samples of SF and ISF from different regions (OC1, OC2, IC) of the lens are shown in Venn diagrams (A, B). Scatterplots of average protein intensities from four biological replicates are shown in $\mathbf{C}$ (OC1 versus OC2) and D (OC2 versus IC).

dramatic fiber cell proteome changes can be seen across these three regions that were derived from a narrow zone of the lens cortex. These dramatic proteome changes can be demonstrated by scatterplots (Figs. 2C, 2D) and Pearson correlations of protein signal intensities (Supplementary Fig. S1). Pearson correlations between samples from different regions were consistently lower than correlations between biological replicates. Comparing the proteomes of SF and ISF from OC2 to OC1, scatterplots for average protein intensities of four biological replicates in different regions of the lens showed regional proteome changes in both SF and ISF (Fig. 2C). Comparing the OC2 to the IC region, the $R^{2}$ value was greater than 0.94 , indicating few proteome changes in SF (Fig. 2D), but the ISF proteome showed more changes, indicating dramatic changes of membrane and membrane-associated proteins from the OC2 to IC region.

To study protein expression changes in the different regions of the lens, four Welch tests were performed (SF in OC1 versus $\mathrm{SF}$ in OC2, ISF in OC1 versus ISF in OC2, $\mathrm{SF}$ in OC2 versus $\mathrm{SF}$ in IC, and ISF in OC2 versus ISF in IC). The results from four Welch tests are shown in Supplementary Table S1. A comparison of OC1 and OC2 results identified 234 proteins in SF and 284 proteins in ISF that were downregulated in OC2. Eighty-nine proteins in SF and 154 proteins in ISF were upregulated in OC2 compared with OC1. When comparing IC with OC2, 74 proteins in SF and 332 proteins in ISF were downregulated in IC, and 61 proteins in SF and 125 proteins in ISF were upregulated in IC. Hierarchical clustering was then performed for statistically significantly changed proteins between regions. The results can be visualized by heatmaps generated during clustering (Fig. 3), and detailed information is included in Supplementary Table S1. Figures 3A and $3 \mathrm{~B}$ show the heatmaps generated from comparisons of ISF from different regions. As expected, the resulting
A

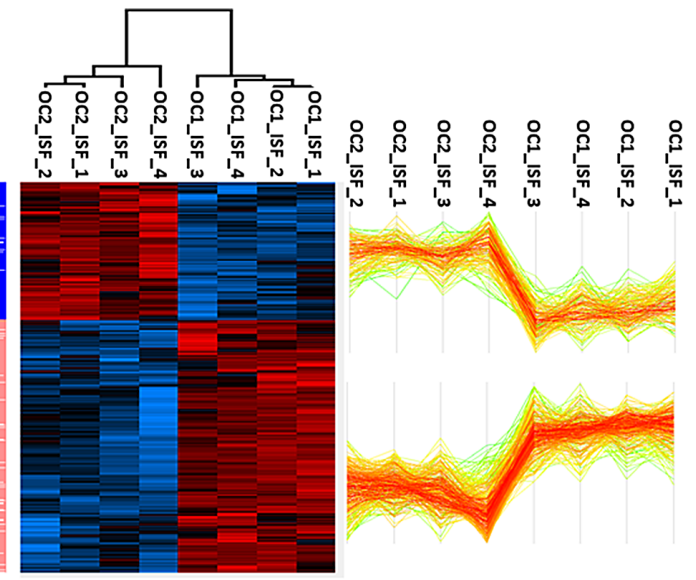

B
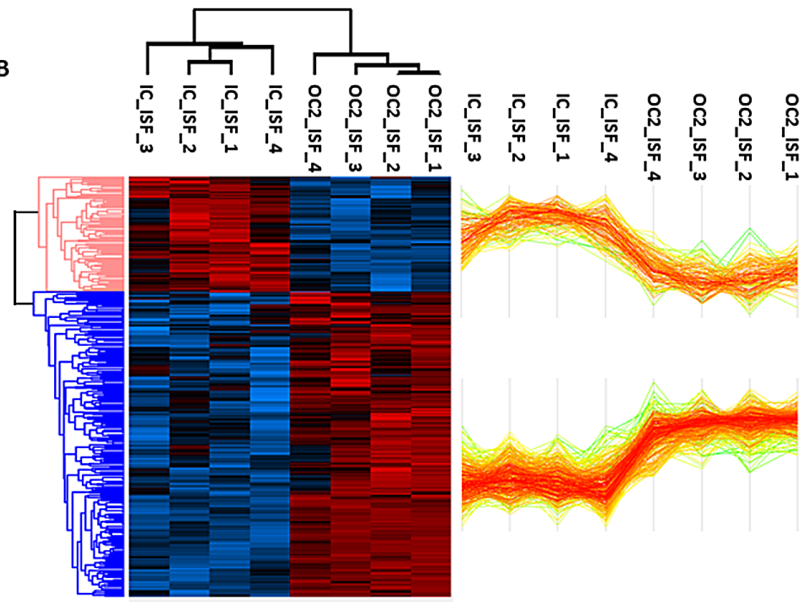

Figure 3. Heatmap and hierarchical clustering of identified statistically significant changed proteins between different regions in ISF. Welch $t$-test and hierarchical clustering were performed by Perseus based on Euclidean distance of statistically significant changed proteins $(P<0.05)$. Hierarchical clustering defined two clusters of proteins for each comparison (A: comparing OC1 and OC2; B: comparing OC2 and IC). Profile plots shown on the right illustrate each protein intensity changes across all samples for a specific cluster.

dendrogram segregated different regions, and four biological replicates clustered together. Therefore, these differentially expressed proteins provide molecular signatures that distinguish different regions of the lens cortex. Similar heatmaps for proteins differentially expressed between regions in SF can be found in Supplementary Figure S2.

To visualize proteins that show large-magnitude changes with high statistical significance, volcano plots for ISF samples are shown in Figure 4. Corresponding volcano plots for SF samples can be found in Supplementary Figure S3. From OC1 to OC2, proteins showing a large fold increase or decrease can be detected (Fig. 4A), whereas from OC2 to IC, many proteins significantly decrease in abundance and very few proteins dramatically increase abundance, suggesting protein degradation in the IC region (Fig. $4 \mathrm{~B}$ ). Proteins that show large fold changes include cell-cell junctional proteins, cytoskeletal proteins, membrane proteins, and some organelle-related proteins. The volcano plots show three proteins that are dramatically more abundant in the OC2 region compared to the OC1 region. These three proteins include lengsin (LGSN), chloride intracellular 

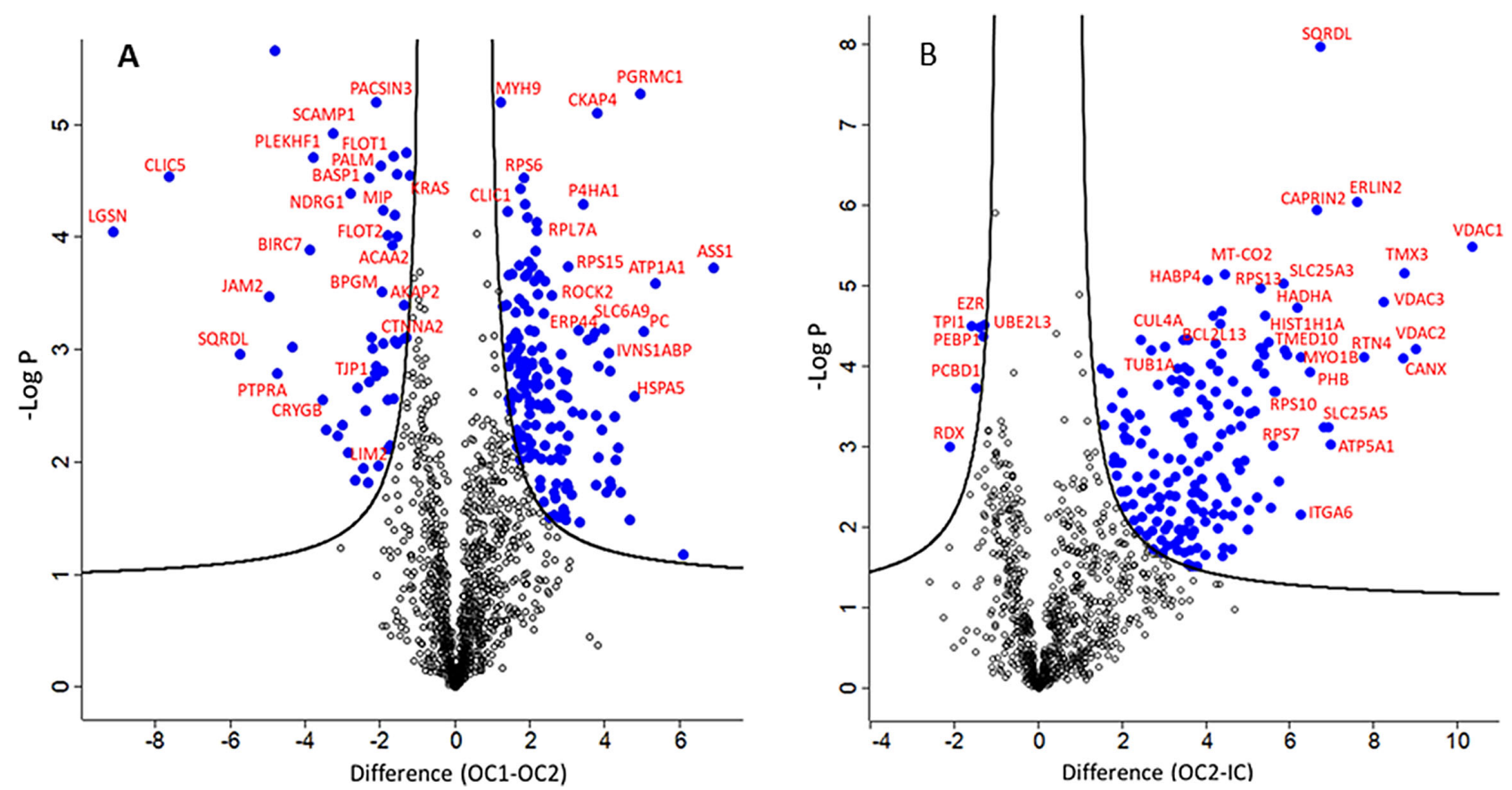

FigURE 4. Volcano plots of spatially differentially expressed proteins measured in ISF. Proteins were graphed by fold change (difference) on the $\mathrm{x}$-axis and significance $(-\log \mathrm{p})$ on the $\mathrm{y}$-axis using a false discovery rate of 0.01 and an $\mathrm{S} 0$ of 0.5 . Statistical analysis was performed in Perseus. Blue circles represent proteins that are significantly changed between OC1 and OC2 (A) or OC2 and IC (B). Due to sequence homology, $\gamma \mathrm{B}, \gamma \mathrm{C}$, and $\gamma \mathrm{D}$ were combined and shown as CRYGB.

channel protein 5 (CLIC5), and lactase-like protein (LCTL). Increasing expression of these proteins was detected in both SF (Supplementary Fig. S3A) and ISF (Fig. 4A). The abundance of these proteins was maintained in the IC region and did not show a significant change in the IC region compared to the OC2 region. Increased levels of CLIC5 were accompanied by a significant decrease of another chloride intracellular channel protein, CLIC1, indicating a transition to a different chloride channel protein in fiber cells.

GO enrichment analysis of differentially represented proteins against the background of all quantified proteins identified GO terms and pathways that are over- or underrepresented in each region (Figs. 5, 6). As expected, in OC2, lens fiber cells have started to remove cellular organelles and reduce protein synthesis. GO terms such as ribosome, translation, and endoplasmic reticulum (ER) lumen had lower representation in the OC2 region compared to the OC1 region. Many proteins involved in these processes were not detected in IC samples or showed a downward trend in IC samples. Unlike ER and ribosomal proteins, mitochondrial proteins were not downregulated in OC2. Mitochondrial proteins did not decrease until cells were in the IC region. Many mitochondrial proteins increased in OC2 samples compared to OC1 samples, including proteins related to $\beta$-oxidation (HADHA, HADHB, ETFDH) and some mitochondrial channel proteins (VDAC1, VDAC2, VDAC3, SLC25A13). Other than these expected protein changes related to organelle degradation, one overrepresented pathway in OC2 samples is related to the proteasome and ubiquitination. This process was found to be further increased in IC samples. Glycolysis is overrepresented in IC samples due to upregulation of many enzymes involved in the glycolysis pathway (Supplementary Table S1). Another notable feature related to fiber cells in the IC region is the decrease of struc- tural constituent cytoskeleton and microtubule, including filamins, spectrins, vimentin and tubulins, and so on. Downregulation of many cytoskeletal proteins was accompanied with upregulation of several actin binding proteins such as actin-related protein $2 / 3$ complex subunit $1 \mathrm{~A}$ and 3 (ARPC1A and ARPC3), F-actin-capping protein subunit $\beta$ (CAPZB), ezrin, and radixin. Interestingly, glutathione synthase was found to be consistently increased in both SF and ISF in OC2 samples compared with OC1 samples and continued to increase in samples from the IC region. Another essential enzyme for glutathione (GSH) synthesis, glutamate-cysteine ligase catalytic subunit, did not significantly change between regions. The biological process related to reactive oxygen species is significantly increased in IC samples, including increased abundance of families of peroxiredoxin and glutathione S-transferase that could consume the reduced form of GSH. This result suggests that fiber cells may adapt a high GSH synthesis ability in the barrier region to fulfill the large GSH requirement of the lens.

\section{The Closing of Extracellular Diffusion Is Accompanied by Changing of Junction Protein Expression}

A major goal of this study is to understand the molecular signatures that contribute to the closing of the extracellular space. Cell-cell junctions affect extracellular space and participate in paracellular barrier formation ${ }^{28}$; therefore, proteins that are involved in cell-cell junctions were analyzed in captured lens regions. The results show that junction proteins are among the proteins that undergo significant changes between regions, including proteins involved 
A

B

(GOCC) small ribosomal subunit

(GOBP) regulation of cellular am ino acid metabolic process (GOBP) negative regulation of ubiquitin-protein ligase activity (GOBP) protein polyubiquitination (KEGG) Proteasome

(GOBP) regulation of small GTPase mediated signal transduction (GOBP) regulation of protein polymerization (GOBP) proteasomal ubiquitin-independent protein catabolic process (GOCC) proteasome core complex (GOMF) threonine-type peptidase activity (GOMF) wide pore channel activity

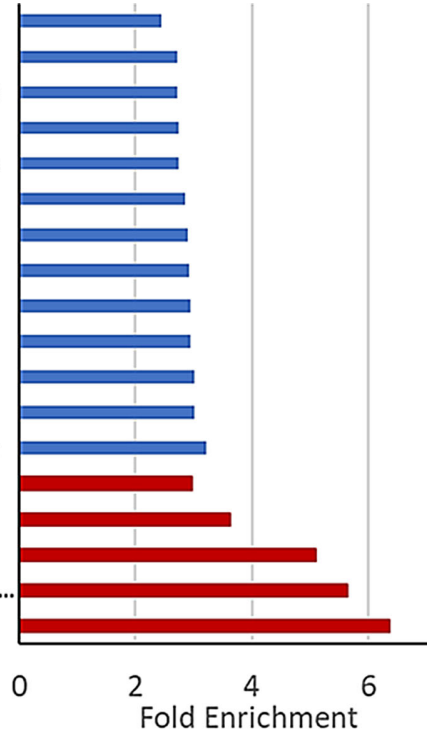

Fold Enrichment

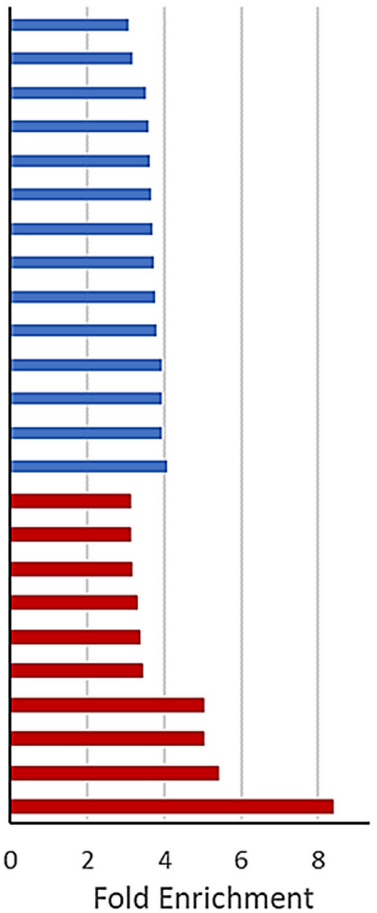

Figure 5. GO terms and pathway enrichment analysis for differentially expressed proteins in OC2 compared with OC1. Enriched GO terms and pathways were analyzed using a Fisher exact test with a Benjamini-Hochberg FDR of 0.05 and total quantified proteins as background. Only GO terms with fold change greater than 2 are shown. When a parent term is present, the child term is not included. Blue and red bars represent GO terms and pathways that are decreased or increased, respectively, in OC2 compared with OC1. (A) Enriched terms in SF. (B) Enriched terms in ISF.

in adherens junctions, tight junctions, gap junctions, and square array junctions.

Adherens junctions and associated actin microfilaments are believed to be involved in stabilizing the structural integrity of lens cells during accommodation and in preserving a specific lens shape. ${ }^{29}$ Major adherens junction proteins, such as $\mathrm{N}$-cadherin $(\mathrm{CDH} 2)$, catenin $\beta 1$ (CTNNB1), and Armadillo repeat protein deleted in velocardio-facial syndrome (ARVCF), did not show statistically significant changes from OC1 to OC2 regions (Fig. 7A). However, a significant decrease of catenin $\alpha 1$ (CTNNA1) in
SF and a significant increase of catenin $\alpha 2$ (CTNNA2) in ISF were detected in the OC2 region compared with the OC1 region (Supplementary Table S1). The increase of CTNNA2 and decrease of CTNNA1 are consistent with the transition from $\alpha \mathrm{E}$-catenin (epithelial type) to $\alpha \mathrm{N}$-catenin (neuronal type) with fiber cell differentiation reported previously. ${ }^{30}$ Comparing the IC region with the OC2 region, all major adherens junction proteins were significantly decreased in the IC region (Fig. 7D).

Another group of proteins that was significantly changed across the three regions is tight junction associated proteins. 

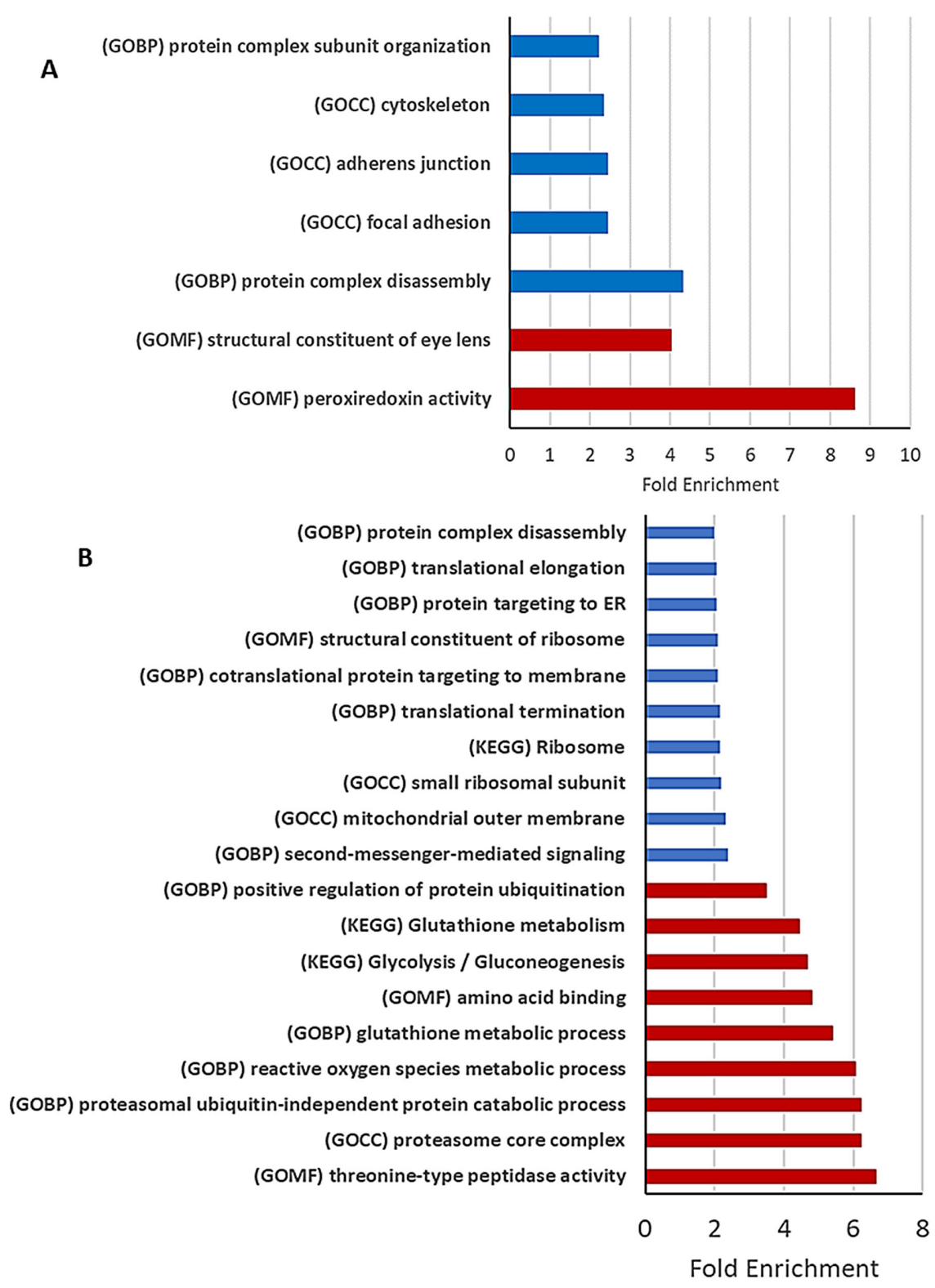

FiguRE 6. GO terms and pathway enrichment analysis for differentially expressed proteins in IC compared with OC2. Enriched GO terms and pathways were analyzed using a Fisher exact test with Benjamini-Hochberg FDR of 0.05 and total quantified proteins as background. Only GO terms with fold change greater than 2 are shown. When a parent term is present, the child term is not included. Blue and red bars represent GO terms and pathways that are decreased or increased, respectively, in IC compared with OC2. (A) Enriched terms in SF. (B) Enriched terms in ISF

Several well-known tight junction proteins were detected in lens fiber cells, including tight junction protein ZO-1 (TJP1), junctional adhesion molecule 2 (JAM2), and coxsackievirus and adenovirus receptor homolog (CXADR). These proteins were significantly increased in OC2 samples compared to OC1 samples and tended to decrease in IC samples even though some changes did not reach statistical significance (Fig. 4, Figs. 7B, 7E). Two PMP-22/EMP/MP20/claudin family proteins, lens membrane intrinsic protein (Lim2) and TMEM47, are present in the lens fiber cells. ${ }^{17}$ Similar to tight junction proteins, $\operatorname{Lim} 2$ was found to be significantly increased in OC2 samples and decreased in IC samples. TMEM47 was increased in OC2 samples and maintained similar levels in IC samples. Similarly, gap junction proteins connexin 50 (GJA8) and connexin 46 (GJA3) increased in
OC2 samples compared with OC1 samples and did not show significant changes between OC2 and IC regions.

An additional cell junction type, square array junctions, are present in the deep cortex and mature lens fibers and are found to be especially rich in AQP0 and its cleavage products. $^{20,21,31}$ Our results show that AQP0 was significantly increased in OC2 samples compared to OC1 samples (Fig. 4A, Fig. 7C) and continued increasing in IC samples compared to OC2 samples (Supplementary Table S1, Fig. 7F). As shown in Figures $4 \mathrm{~B}$ and $7 \mathrm{~F}$, along with increased AQP0 in ISF samples, ezrin and radixin are also significantly increased in IC samples, both of which have been shown to interact with AQP0. ${ }^{32}$ The increased ezrin in the ISF was also accompanied with decreased ezrin in the SF (Supplementary Table S1). 

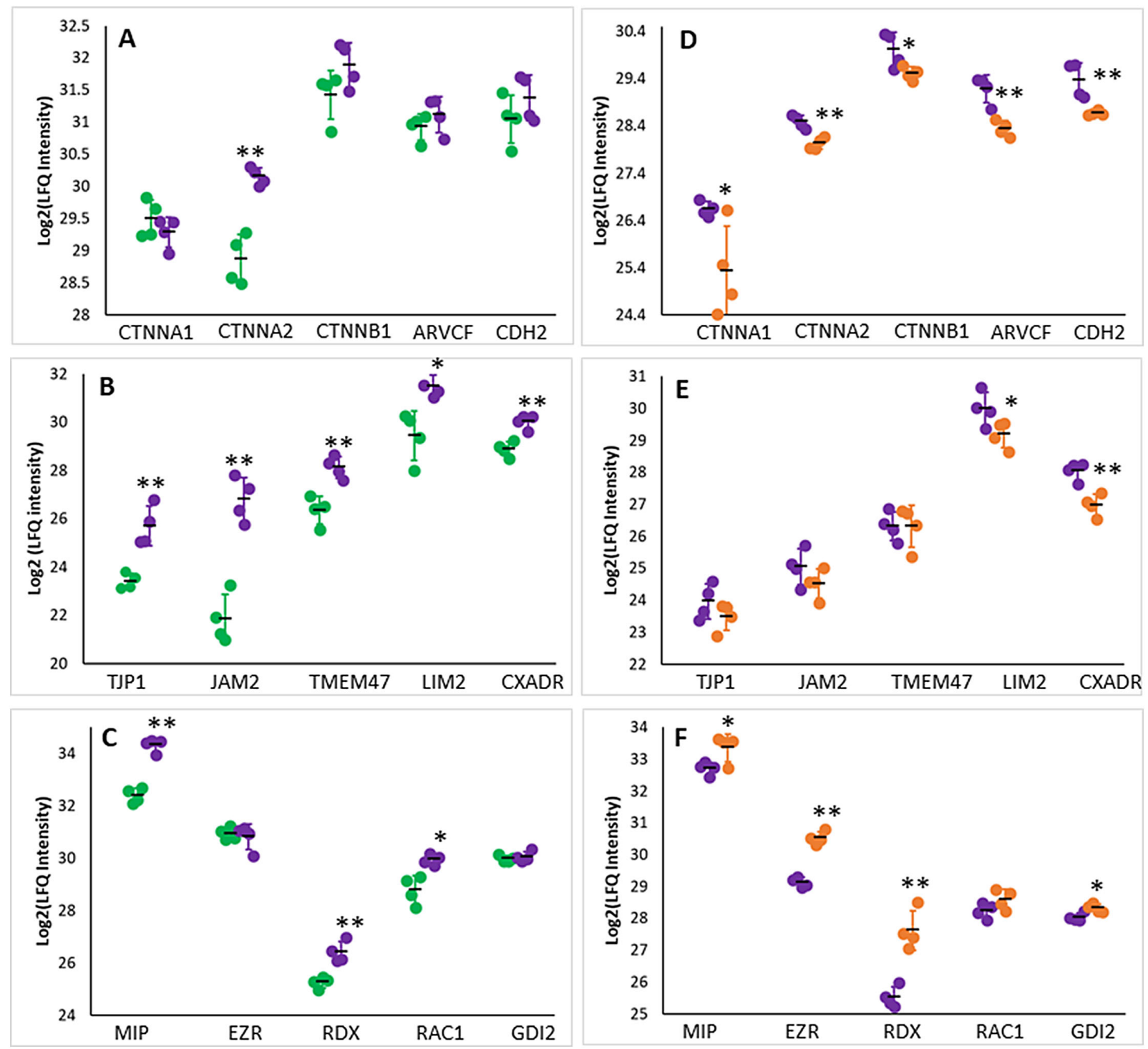

Figure 7. Protein LFQ intensity of major junctional proteins. LFQ intensities of major cell junction-related proteins are plotted. (A-C) Comparison of OC2 region with OC1 region. (D-F) Comparison of IC region with OC2 region. Green: OC1; purple: OC2; orange: IC. $* P<0.05$. *** $P<0.01$.

A protein-protein interaction network was constructed using String analysis ${ }^{27}$ for proteins that have the same expression trends in each lens region, and protein interaction networks that are connected with cell junction proteins were extracted and are shown in Figure 8. This network analysis shows highly connected nodes with junction proteins, including proteins that regulate or associate with the actin cytoskeleton and GTPases and their regulators. Adherens junction and tight junction proteins were clustered together through TJP1, which is also linked to gap junctions through connexins. These junction proteins are connected with the actin cytoskeleton network and the small GTPase signal transduction network (Figs. 8A, 8B). Together with the downregulation of adherens junction and tight junction proteins in the IC region, many actin binding cytoskeleton proteins are downregulated. The spectrin-ankyrin network is also linked to the adherens junction and tight junction network and showed a consistent trend of changing with these junction networks.
Figure 8B also shows proteins associated with focal adhesions (cell-matrix adhesions) such as ITGB1, ITGA6, TLN1, FLNC, and FLNB decreased in the IC region. The decrease of ezrin/periplakin/periaxin/desmoyokin (EPPD) complex proteins in the IC region can also be seen in Figure $8 \mathrm{~B}$. Among the proteins increased in the IC region, along with AQP0 and ezrin/radixin/moesin (ERM) proteins, were proteins involved in regulation of the actin cytoskeleton (ARPC1A, ARPC3, MAPK1, and GSN), proteins belonging to the 14-3-3 family (YWHAE, YWHAG, and YWHAE), the small GTPase family (RAN, RHEB, and RAB6B), and the GTPbinding protein, ARL3.

\section{Discussion}

In this study, we measured differential protein expression across three narrow regions in the cortex of lens equatorial sections to characterize proteins that could be involved in closing lens extracellular space and forming an extracellular 

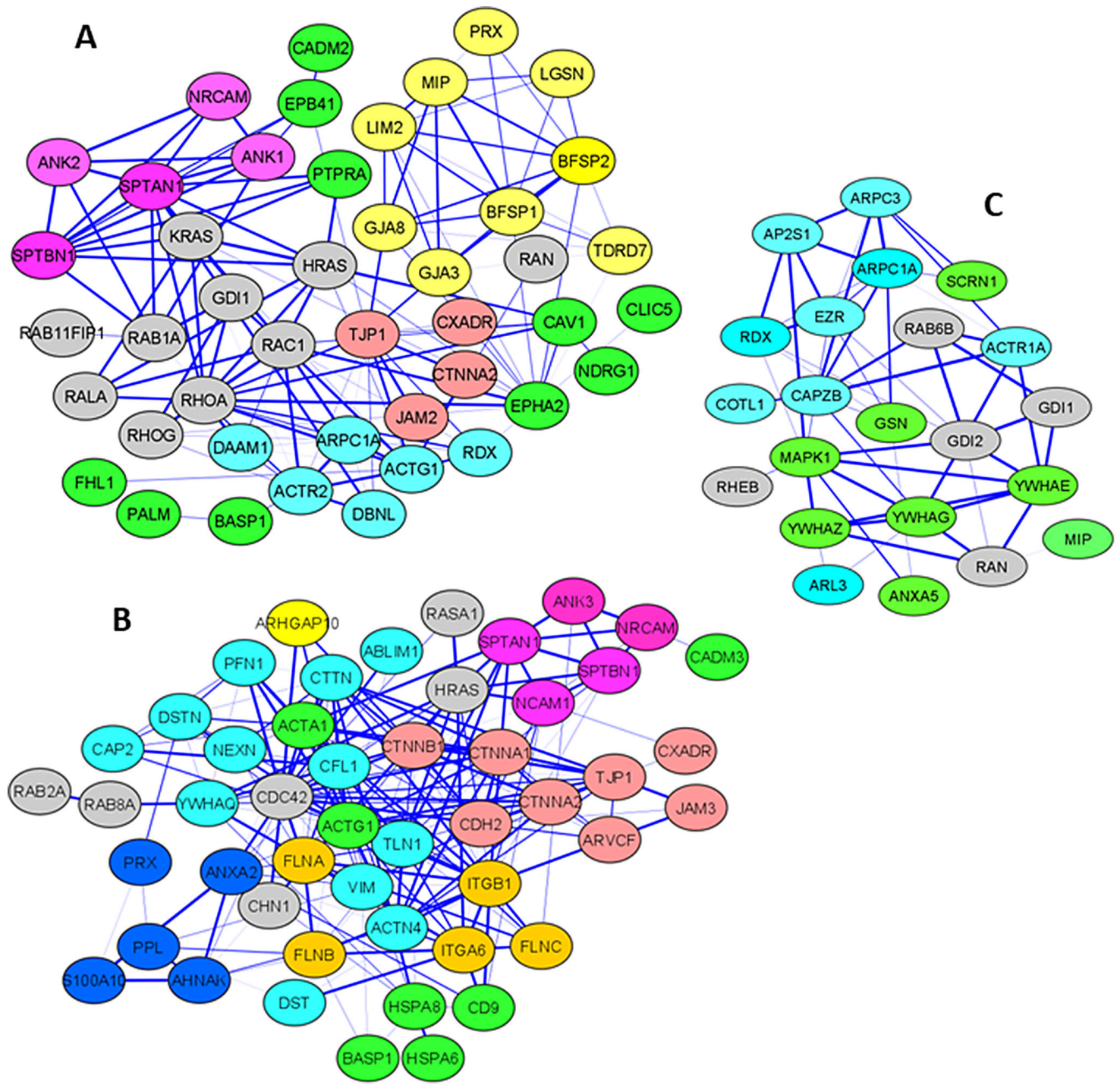

FIGURE 8. Protein-protein network analysis. Intermolecular interactions of differentially expressed proteins that are associated with cell junction and actin cytoskeleton were generated by STRING 11.0. The minimum required interaction score was medium confidence of 0.400 . Nodes represent proteins; same-node colors indicate membership of the same cluster, and line thickness denotes the confidence level of the interaction. (A) Proteins increased in OC2 compared with OC1. (B) Proteins decreased in IC compared with OC2. (C) Proteins increased in IC compared with OC2.

diffusion barrier. Our results suggest that the closing of the extracellular space is a two-step process whereby closing of the broad side spacing of fiber cells (OC2 region) is followed by closing of the short side spacing of fiber cells (IC region). Coinciding with changing of extracellular space, cellular morphology and protein expression change dramatically. Cell-cell adhesion proteins are believed to play important roles in regulating the extracellular space, and our results demonstrate that junction proteins such as adherens junction proteins, tight junction-related proteins, gap junction proteins, and lens fiber membrane intrinsic protein (LIM2) increase in the region where extracellular space between the broad sides of lens fiber cells is reduced. Further experiments are needed to determine whether these proteins are involved in closing the extracellular space of the broad sides of fiber cells.

Based on previous reports, adherens junctions can be found at the narrow faces of the fiber cell, especially in the "intersections" where three hexagonal fiber cells meet. ${ }^{16,33}$ Adherens junctions are not believed to be involved in the closing extracellular diffusion along the broad sides of the fiber cells. Adherens junctions and associated actin microfilaments are believed to be involved in stabilizing the structural integrity of lens cells during accommodation and in preserving a specific lens shape. ${ }^{29}$ Other than the switch of CTNNA1 to CTNNA2, we did not detect significant changes of major adherens junction proteins. Gap junctions can be found in both broad sides and narrow sides of the fiber cells ${ }^{19}$ and have been reported to restrict the passage of large solutes through extracellular space.? In addition, connexin 50 can function as an adhesive molecule, and $\mathrm{Cx} 50 \mathrm{KO}$ lenses have exhibited increased extracellular space. ${ }^{34}$ Therefore, the increase in gap junction proteins in the OC2 region could contribute to the restriction of dye penetration between the broad sides of fiber cells. 
Other proteins that could be involved in the narrowing of the broad side extracellular space include LIM2 and several tight junction-related proteins, including ZO-1, JAM2 and JAM3, and CXADR that are all increased in the OC2 region. The transition of $\mathrm{ZO}-1$ from the narrow faces of the fiber cells in outer cortex to the broad faces of the fiber cells in the inner cortex has been reported. ${ }^{35}$ Due to the lack of occludins and claudins, tight junctions are not believed to exist in lens fiber cells ${ }^{14,16}$; however, the tight junction protein $\mathrm{ZO}-1$ has been reported to interact with lens connexins. ${ }^{35}$ In addition, the increase of $\mathrm{ZO}-1$ in the OC2 region coincided with an increase of LIM2, a PMP22/EMP/MP20/claudin family member. ${ }^{17}$ The cell adhesive property of LIM2 has been reported, ${ }^{36}$ and insertion of LIM2 into lens fiber cell membranes was found to correlate with the formation of an extracellular diffusion barrier. ${ }^{10}$ Therefore, LIM2 could contribute to the narrowing of extracellular space; however, whether LIM2 interacts with ZO-1 to form a tight junction-like structure is unknown.

In addition to cell-cell adhesion proteins, another important protein that could be involved in closing the extracellular space is lens major membrane protein, AQP0. Our results indicate that AQP0 dramatically increased from the OC1 region to the OC2 region and is one of the very few junction proteins that further increased in the IC region where extracellular diffusion is completely stopped. Previously, AQP0 was found to redistribute throughout the cell membrane in nonnucleated fiber cells from plaque-like structures predominantly on the broad sides in nucleated fiber cells. ${ }^{37}$ The transition of a certain portion of AQP0 to the narrow sides of the fiber cells could play a key role in closing the extracellular space between the narrow sides since the cell-cell adhesion function AQP0 has been well documented. ${ }^{38,39}$

The localization and distribution of membrane proteins in the plasma membrane are regulated through the membraneassociated cytoskeleton. ${ }^{40}$ Previously, AQP0 was found to interact with ERM proteins, ${ }^{32}$ proteins that are linkers of the plasma membrane and the cortical actin cytoskeleton. In this study, ERM proteins ezrin and radixin are among the very few proteins that increase in association with membrane proteins in the IC region. Interactions between AQP0 and ERM proteins could play a role in driving the redistribution of AQP0 in fiber cell membranes. Ezrin is one of the components of the EPPD complex along with periplakin, periaxin, and desmoyokin. ${ }^{16}$ Our results also show periplakin and desmoyokin (AHNAK) abundances decreased significantly in the IC region, suggesting the decomposition of EPPD complexes. We hypothesize that ezrin released from EPPD complex redistributes in the membrane and interacts with AQP0. A clear transition from soluble fraction to insoluble fraction was detected in the barrier region. Proteins that are involved in this process may also include Rho GTPase and its negative regulators, GDP dissociation inhibitors (GDIs). Our results show GDI2 and RAC1 also increased in the IC region. Previously, reduced Rho GTPase activity disrupted the redistribution of AQP0 in lens fiber cells. ${ }^{41,42}$ The association of ERM proteins and plasma membrane is regulated by the Rho-dependent signaling pathway, ${ }^{43}$ and direct interaction of GDI with ERM proteins has been reported. ${ }^{44}$

The dramatic increase in expression of LGSN, CLIC5, and LCTL suggest important roles for these proteins in lens fiber cells. Lengsin was reported to colocalize with lens intermediate filament proteins in maturing fiber cells and may act as a component of the cytoskeleton itself or as a chaperone for the reorganization of intermediate filaments. ${ }^{45}$ A circulating flux of $\mathrm{Cl}^{-}$ions mediated by an unknown chloride channel is believed to be involved in the operation of the lens microcirculation system, and a chloride channel inhibitor causes extracellular dilation. ${ }^{46}$ We predict the unidentified chloride channel reported by Webb et al. $^{46}$ is CLIC5. Even though CLIC1 was also detected, its abundance was significantly decreased in maturing fiber cells. The exchange of chloride channels suggests that CLIC5 must play a particular role in the lens. The function of chloride channels in the lens may include maintenance of a steady hydrated state and cell volume, regulation of $\mathrm{pH}$, and maintenance of a circulating flux of $\mathrm{Cl}^{-}$ions. ${ }^{46}$ LCTL was found to be essential for CLIC5 expression. ${ }^{47}$ In addition, LCTL is highly expressed in the lens and was reported to regulate suture formation. ${ }^{47}$ Therefore, despite direct experimental evidence, we hypothesize that these proteins could play important roles in lens microcirculation system.

In addition to barrier-related proteome changes, this study also identifies protein changes associated with some important biological processes involved in fiber cell differentiation such as organelle degradation, cytoskeletal organization, and cellular metabolism. These results will be the focus of a separate report. Lastly, although the focus of this work is on proteome changes in the barrier region, it is important to note that plasma membrane cholesterol and phospholipids are enriched at fiber cell junctions ${ }^{48}$ and that plasma membrane lipid composition can affect membrane protein structure and function ${ }^{49}$ as well as plasma membrane permeability to polar solutes. ${ }^{50}$ Given that cholesterol concentration increases from the outer cortex to the nucleus ${ }^{51}$ and that the high cholesterol content in the lens results in hydrophobic cholesterol domains, ${ }^{52}$ it is conceivable that cholesterol could play a role in barrier formation.

The current study identifies potential proteins and biological processes that could be involved in regulating the extracellular space of lens fiber cells. Further experiments are needed to confirm the function of these proteins in regulating lens microcirculation system. Furthermore, our current study only focused on a narrow region in the equator of the lens. This study, therefore, does not examine proteome changes between the barrier region and the nucleus region and the proteome changes along the fiber cell length from anterior to posterior poles. Further comparisons between all regions are expected to provide a more complete picture of lens spatially resolved proteome changes that will help us to understand more about the lens microcirculation system and lens homeostasis.

\section{Acknowledgments}

Supported by NIH Grants R01 EY013462 and P30 EY008126.

Disclosure: Z. Wang, None; L.S. Cantrell, None; K.L. Schey, None

\section{References}

1. Mathias RT, Rae JL, Baldo GJ. Physiological properties of the normal lens. Physiol Rev. 1997;77:21-50.

2. Mathias RT. Steady-state voltages, ion fluxes, and volume regulation in syncytial tissues. Biophys J. 1985;48:435-448.

3. Vaghefi E, Donaldson PJ. The lens internal microcirculation system delivers solutes to the lens core faster than would 
be predicted by passive diffusion. Am J Physiol Regul Integr Comp Physiol. 2018;315:R994-R1002.

4. Donaldson P, Kistler J, Mathias RT. Molecular solutions to mammalian lens transparency. News Physiol Sci. 2001;16:118-123.

5. Unakar NJ, Tsui JY. Sodium-potassium-dependent ATPase: I. Cytochemical localization in normal and cataractous rat lenses. Invest Ophthalmol Vis Sci. 1980;19:630-641.

6. Delamere NA, Dean WL. Distribution of lens sodiumpotassium-adenosine triphosphatase. Invest Ophthalmol Vis Sci. 1993;34:2159-2163.

7. Baldo GJ, Mathias RT. Spatial variations in membrane properties in the intact rat lens. Biophys J. 1992;63:518-529.

8. Parmelee JT. Measurement of steady currents around the frog lens. Exp Eye Res. 1986;42:433-441.

9. Vaghefi E, Walker K, Pontre BP, Jacobs MD, Donaldson PJ. Magnetic resonance and confocal imaging of solute penetration into the lens reveals a zone of restricted extracellular space diffusion. Am J Physiol Regul Integr Comp Physiol. 2012;302:R1250-R1259.

10. Grey AC, Jacobs MD, Gonen T, Kistler J, Donaldson PJ. Insertion of MP20 into lens fibre cell plasma membranes correlates with the formation of an extracellular diffusion barrier. Exp Eye Res. 2003;77:567-574.

11. Lim JC, Walker KL, Sherwin T, Schey KL, Donaldson PJ. Confocal microscopy reveals zones of membrane remodeling in the outer cortex of the human lens. Invest Ophthalmol Vis Sci. 2009;50:4304-4310.

12. Cohen AI, The electron microscopy of the normal human lens. Invest Ophthalmol. 1965;4:433-446.

13. Rodgers LS, Beam MT, Anderson JM, Fanning AS. Epithelial barrier assembly requires coordinated activity of multiple domains of the tight junction protein ZO-1. J Cell Sci. 2013;126:1565-1575.

14. Bassnett S, Wilmarth PA, David LL. The membrane proteome of the mouse lens fiber cell. Mol Vis. 2009;15:2448-2463.

15. Wang Z, Han J, David LL, Schey KL. Proteomics and phosphoproteomics analysis of human lens fiber cell membranes. Invest Ophthalmol Vis Sci. 2013;54:1135-1143.

16. Straub BK, Boda J, Kuhn C, et al. A novel cell-cell junction system: the cortex adhaerens mosaic of lens fiber cells. $J$ Cell Sci. 2003;116:4985-4995.

17. Van Itallie CM, Anderson JM. The molecular physiology of tight junction pores. Physiology. 2004;19:331-338.

18. Shiels A, King JM, Mackay DS, Bassnett S. Refractive defects and cataracts in mice lacking lens intrinsic membrane protein-2. Invest Ophthalmol Vis Sci. 2007;48:500508.

19. Tenbroek E, Arneson M, Jarvis J, Louis C. The distribution of the fiber cell intrinsic membrane proteins MP20 and connexin46 in the bovine lens. J Cell Sci. 1992;103:245257.

20. Costello MJ, McIntosh TJ, Robertson JD. Distribution of gap junctions and square array junctions in the mammalian lens. Invest Ophthalmol Vis Sci. 1989;30:975-989.

21. Lo WK, Harding CV. Square arrays and their role in ridge formation in human lens fibers. $J$ Ultrastruct Res. 1984;86:228-245.

22. Zampighi G, Simon SA, Robertson JD, McIntosh TJ, Costello MJ. On the structural organization of isolated bovine lens fiber junctions. J Cell Biol. 1982;93:175-189.

23. Rappsilber J, Mann M, Ishihama Y. Protocol for micropurification, enrichment, pre-fractionation and storage of peptides for proteomics using StageTips. Nat Protoc. 2007;2:1896-1906.

24. Cox J, Hein MY, Luber CA, Paron I, Nagaraj N, Mann M. Accurate proteome-wide label-free quantification by delayed normalization and maximal peptide ratio extrac- tion, termed MaxLFQ. Mol Cell Proteomics. 2014;13:25132526.

25. Cox J, Neuhauser N, Michalski A, Scheltema RA, Olsen JV, Mann M. Andromeda: a peptide search engine integrated into the MaxQuant environment. J Proteome Res. 2011;10:1794-1805.

26. Tyanova $S$, Temu T, Sinitcyn $P$, et al. The Perseus computational platform for comprehensive analysis of (prote)omics data. Nat Methods. 2016;13:731-740.

27. Snel B, Lehmann G, Bork P, Huynen MA. STRING: a webserver to retrieve and display the repeatedly occurring neighbourhood of a gene. Nucleic Acids Res. 2000;28:34423444.

28. Garcia MA, Nelson WJ, Chavez N. Cell-cell junctions organize structural and signaling networks. Cold Spring Harb Perspect Biol. 2018;10:a029181.

29. Lo W-K. Adherens junctions between cortical fiber cells of the ocular lens. Invest Ophthamol Vis Sc. 1987;28(suppl): 83.

30. Maddala R, Rao PV. Switching of $\alpha$-catenin from epithelial to neuronal type during lens epithelial cell differentiation. Invest Ophthalmol Vis Sci. 2017;58:3445-3455.

31. Gonen T, Sliz P, Kistler J, Cheng Y, Walz T. Aquaporin-0 membrane junctions reveal the structure of a closed water pore. Nature. 2004;429:193-197.

32. Wang Z, Schey KL. Aquaporin-0 interacts with the FERM domain of ezrin/radixin/moesin proteins in the ocular lens. Invest Ophthalmol Vis Sci. 2011;52:5079-5087.

33. Lo WK. Adherens junctions in the ocular lens of various species: ultrastructural analysis with an improved fixation. Cell Tissue Res. 1988;254:31-40.

34. Hu Z, Shi W, Riquelme MA, et al. Connexin 50 functions as an adhesive molecule and promotes lens cell differentiation. Sci Rep. 2017;7:5298.

35. Nielsen PA, Baruch A, Shestopalov VI, et al. Lens connexins alpha3Cx46 and alpha8Cx 50 interact with zonula occludens protein-1 (ZO-1). Mol Biol Cell. 2003;14:24702481.

36. Gonen T, Donaldson P, Kistler J. Galectin-3 is associated with the plasma membrane of lens fiber cells. Invest Ophthalmol Vis Sci. 2000;41:199-203.

37. Grey AC, Li L, Jacobs MD, Schey KL, Donaldson PJ. Differentiation-dependent modification and subcellular distribution of aquaporin-0 suggests multiple functional roles in the rat lens. Differentiation. 2009;77:70-83.

38. Kumari SS, Varadaraj K. Intact AQP0 performs cell-to-cell adhesion. Biochem Biophys Res Commun. 2009;390:10341039.

39. Varadaraj K, Kumari SS. Molecular mechanism of aquaporin 0 -induced fiber cell to fiber cell adhesion in the eye lens. Biochem Biophys Res Commun. 2018;506:284-289.

40. Sako Y, Kusumi A. Compartmentalized structure of the plasma membrane for receptor movements as revealed by a nanometer-level motion analysis. J Cell Biol. 1994;125:12511264.

41. Maddala R, Deng PF, Costello JM, Wawrousek EF, Zigler JS, Rao VP. Impaired cytoskeletal organization and membrane integrity in lens fibers of a Rho GTPase functional knockout transgenic mouse. Lab Invest. 2004;84:679-692.

42. Maddala R, Reneker LW, Pendurthi B, Rao PV. Rho GDP dissociation inhibitor-mediated disruption of Rho GTPase activity impairs lens fiber cell migration, elongation and survival. Dev Biol. 2008;315:217-231.

43. Hirao M, Sato N, Kondo T, et al. Regulation mechanism of ERM (ezrin/radixin/moesin) protein/plasma membrane association: possible involvement of phosphatidylinositol turnover and rho-dependent signaling pathway. J Cell Biol. 1996;135:37-51. 
44. Takahashi K, Sasaki T, Mammoto A, et al. Direct interaction of the rho GDP dissociation inhibitor with ezrin/radixin/moesin initiates the activation of the rho small G protein. J Biol Chem. 1997;272:23371-23375.

45. Wyatt K, Gao C, Tsai J, Fariss RN, Ray S, Wistow G. A role for lengsin, a recruited enzyme, in terminal differentiation in the vertebrate lens. J Biol Chem. 2008;283:66076615.

46. Webb KF, Merriman-Smith BR, Stobie JK, Kistler J, Donaldson $\mathrm{PJ} . \mathrm{Cl}^{-}$influx into rat cortical lens fiber cells is mediated by a $\mathrm{Cl}$ conductance that is not ClC-2 or -3 . Invest Ophthalmol Vis Sci. 2004;45:4400-4408.

47. Fan J, Lerner J, Wyatt MK, et al. The klotho-related protein KLPH (lctl) has preferred expression in lens and is essential for expression of clic 5 and normal lens suture formation. Exp Eye Res. 2018;169:111-121.
48. Fleschner CR, Cenedella RJ. Lipid composition of lens plasma membrane fractions enriched in fiber junctions. $J$ Lipid Res. 1991;32:45-53.

49. Tong J, Canty JT, Briggs MM, McIntosh TJ. The water permeability of lens aquaporin- 0 depends on its lipid bilayer environment. Exp Eye Res. 2013;113:32-40.

50. Mainali L, Raguz M, O'Brien WJ, Subczynski WK. Properties of membranes derived from the total lipids extracted from the human lens cortex and nucleus. Biochim Biophys Acta. 2013;1828:1432-1440.

51. Borchman D, Yappert MC. Lipids and the ocular lens. J Lipid Res. 2010;51:2473-2488.

52. Jacob RF, Cenedella RJ, Mason RP. Direct evidence for immiscible cholesterol domains in human ocular lens fiber cell plasma membranes. J Biol Chem. 1999;274:3161331618. 